\title{
Author index
}

\section{Volume 17}

Alcalde Cuesta, F.,
Lozano Rojo, Á. \&
Vázquez Martínez, A.
C., Insertion-tolerance
and repetitiveness of
random graphs
Andres, J., Randomiza-
tion of Sharkovsky-
type results on the cir-
cle

Backes, L. \& Poletti, M., Continuity of Lyapunov exponents is equivalent to continuity of Oseledets subspaces

Bahlali, K., Gatt, R., Mansouri, B. \& Mtiraoui, A., Backward doubly SDEs and SPDEs with superlinear growth generators

Bao, F., Cao, Y., Han, X. \& Li, J., Efficient particle filtering for stochastic Kortewegde Vries equations

Barrientos, P. G., Fakhari, A., Malicet, D. \& Sarizadeh, A., Expanding actions: Minimality and ergodicity

Bilbao, R. A. \& Oliveira, K., Maximizing entropy measures for random dynamical systems

Bonaccorsi, S. \& Zanella, M., Absolute continuity of the law for solutions of stochastic differential equations with boundary noise

Cao, Y., see Bao
17 (2017) 1750023

17 (2017) 1750017

17 (2017) 1750047

17 (2017) 1750009

17 (2017) 1750008

17 (2017) 1750031

17 (2017) 1750032

17 (2017) 1750045

17 (2017) 1750008
Catuogno, P. J., Ledesma, D. S. \& Ruffino, P. R., Harmonic measures in embedded foliated

da Silva, F. B., see

www.worldscientific.com manifolds

Chen, J. P., Ford, L. Kielty, D., Majumdar, R., McCain, H., O'Connell, D. \& Shum, F. N., Stabilization by noise of a $\mathbb{C}^{2}$-valued coupled system

Choi, S.-Y., see Yoon

Cipriano, I., Entry time statistics to different shrinking sets

Dai, H., see Kong Ledesma

Debs, P. \& Haberkorn, T., Diseases transmission in a $z$-ary tree

Dedecker, J. \& Merlevède, F., A deviation bound for $\alpha$ dependent sequences with applications to intermittent maps

17 (2017) 1750026

17 (2017) 1750046

17 (2017) 1750003

17 (2017) 1750024

17 (2017) 1750015

17 (2017) 1750010

17 (2017) 1750038

17 (2017) 1750005

17 (2017) 1750020

Denker, M. \& Schmalfuß, B., A word from the editors

Deugoué, G., Approximation of the stochastic 2D hydrodynamical type systems driven by non-Gaussian Lévy noise

Fakhari, A., see Barrientos

Fan, X. L. \& Ren, Y., Bismut formulas

17 (2017) 1701001
17 (2017) 1750048

17 (2017) 1750031 
and applications for stochastic (functional) differential equations driven by fractional Brownian motions

Ford, L., see Chen

Gatt, R., see Bahlali

Ge, Q., Li, Z. \& Teng,

Z., Probability analysis of a stochastic SIS epidemic model

Getan, A., Molchanov, S. \& Vainberg, B., Intermittency for branching walks with heavy tails

Gu, A. \& Li, Y., Dynamic behavior of stochastic $p$-Laplacian-type lattice equations

Haberkorn, T., see Debs

Han, X., see Bao

Han, Y., see Yoon

$\mathrm{Hu}, \mathrm{H}$., see Zeng

Ivanov, R. V. \& Temnov, G., Truncated moment-generating functions of the NIG process and their applications

Kielty, D., see Chen

Kim, J.-H., see Yoon

Kong, L. \& Dai, H., Convergence rates in precise asymptotics for a kind of complete moment convergence

Kromer, E., Overbeck, L. \& Röder, J. A. L., Path-dependent BSDEs with jumps and their connection to PPIDEs

Krupski, M., Scaling limits of solutions of linear evolution equations with random initial conditions

Kuhwald, I. \& Pavlyukevich, I., Stochastic resonance with multiplicative heavy-tailed Lévy noise: Optimal tuning on an algebraic time scale

Ledesma, D. S. \& da Silva, F. B., Invariance of 0-currents under diffusions

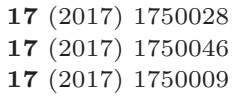

17 (2017) 1750041

17 (2017) 1750044

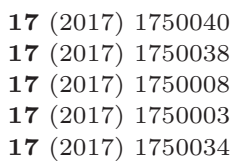

17 (2017) 1750039

17 (2017) 1750046

17 (2017) 1750003

17 (2017) 1750015

17 (2017) 1750036

17 (2017) 1750019

17 (2017) 1750027

17 (2017) 1750010
Ledesma, D. S., see Catuogno

León, J. A. \& Peralta, L., Some Feller and Osgood type criteria for semilinear stochastic differential equations

Li, J., see Bao

Li, X., see Sun

Li, Y., Wang, R., Yao, N. \& Zhang, S., Moderate deviations for a fractional stochastic heat equation with spatially correlated noise

Li, Y., see $\mathrm{Gu}$

$\mathrm{Li}, \mathrm{Z}$., see Ge

Lindner, F., Marheineke, N., Stroot, H., Vibe, A. \& Wegener, R., Stochastic dynamics for inextensible fibers in a spatially semidiscrete setting

Lopes, A. O. \& Mohr, J., Semiclassical limits, Lagrangian states and coboundary equations

Lozano Rojo, Á., see Alcalde Cuesta

Luo, P. \& Tangpi, L., Solvability of coupled FBSDEs with diagonally quadratic generators

Majumdar, R., see Chen Malicet, D., see Barrientos

Mansouri, B., see Bahlali

Marheineke, N., see Lindner

Maticiuc, L., Răşcanu, A. \& Słomiński, L., Multivalued monotone stochastic differential equations with jumps

McCain, H., see Chen

Mengue, J. K. \& Oliveira, E. R., Duality results for iterated function systems with a general family of branches

Merlevède, F., see Dedecker

Mohr, J., see Lopes
17 (2017) 1750026

17 (2017) 1750011

17 (2017) 1750008

17 (2017) 1750033

17 (2017) 1750025

17 (2017) 1750040

17 (2017) 1750041

17 (2017) 1750016

17 (2017) 1750014

17 (2017) 1750023

17 (2017) 1750043

17 (2017) 1750046

17 (2017) 1750031

17 (2017) 1750009

17 (2017) 1750016

17 (2017) 1750018

17 (2017) 1750046

17 (2017) 1750021

17 (2017) 1750005

17 (2017) 1750014 
Molchanov, S., see Getan

Moussaten, S., see Ouahra

Mtiraoui, A., see Bahlali

Muthukumar, P. \& Deepa, R., Infinite horizon optimal control of forwardbackward stochastic system driven by Teugels martingales with Lévy processes

Nakagawa, K., Multifractal rigidity for piecewise linear Markov maps

O'Connell, D., see Chen

Oliveira, E. R., see Mengue

Oliveira, K., see Bilbao

Ouahra, M. A., Moussaten, S. \& Sghir, A., On limit theorems of some extensions of fractional Brownian motion and their additive functionals

Overbeck, L., see Kromer

Pavlyukevich, I., see Kuhwald

Pei, B., see $\mathrm{Xu}$

Peralta, L., see León

Poletti, M., see Backes

Răşcanu, A., see Maticiuc

Ren, Y., see Fan

Röder, J. A. L., see Kromer

Rodrigues, A. \& Zhang, Y., Fair measure and fair entropy for nonMarkov interval maps

Ruffino, P. R., see Catuogno

Russo, F. \& Wurzer, L., Elliptic PDEs with distributional drift and backward SDEs driven by a càdlàg martingale with random terminal time

Salins, M. \& Spiliopoulos, K., Markov processes with spatial delay: Path space characterization, occupation time and properties
17 (2017) 1750044

17 (2017) 1750022

17 (2017) 1750009

17 (2017) 1750020

17 (2017) 1750006

17 (2017) 1750046

17 (2017) 1750021

17 (2017) 1750032

17 (2017) 1750022

17 (2017) 1750036

17 (2017) 1750027

17 (2017) 1750013

17 (2017) 1750011

17 (2017) 1750047

17 (2017) 1750018

17 (2017) 1750028

17 (2017) 1750036

17 (2017) 1750035

17 (2017) 1750026

17 (2017) 1750030

17 (2017) 1750042
Sarizadeh, A., see Barrientos

Schmalfuß, B., see Denker

Sghir, A., see Ouahra

Shum, F. N., see Chen

Słomiński, L., see Maticiuc

Spiliopoulos, K., see Salins

Stroot, H., see Lindner

Sultanov, O., White noise perturbation of locally stable dynamical systems

Sun, X., Li, X. \& Zheng, Y., Governing equations for probability densities of Marcus stochastic differential equations with Lévy noise

Sun, X., Yan, L. \& Zhang, Q., The quadratic covariation for a weighted fractional Brownian motion

Tangpi, L., see Luo

Temnov, G., see Ivanov

Teng, Z., see Ge

Tong, J. \& Zhang, Z., Exponential ergodicity of CIR interest rate model with random switching

Tudor, C. A. \& Xiao, Y., Sample paths of the solution to the fractional-colored stochastic heat equation

Uda, K., Existence of random invariant periodic curves via random semiuniform ergodic theorem

Vainberg, B., see Getan

Vázquez Martínez, A. C., see Alcalde Cuesta

Vibe, A., see Lindner

Wang, R., see Li

Wegener, R., see Lindner

Wu, J.-L., see Xu

Wurzer, L., see Russo

Xiao, Y., see Tudor

$\mathrm{Xu}, \mathrm{Y} .$, Pei, B. \& Wu, J.-L., Stochastic averaging principle for differential equations
17 (2017) 1750031

17 (2017) 1701001

17 (2017) 1750022

17 (2017) 1750046

17 (2017) 1750018

17 (2017) 1750042

17 (2017) 1750016

17 (2017) 1750002

17 (2017) 1750033

17 (2017) 1750029

17 (2017) 1750043

17 (2017) 1750039

17 (2017) 1750041

17 (2017) 1750037

17 (2017) 1750004

17 (2017) 1750007

17 (2017) 1750044

17 (2017) 1750023

17 (2017) 1750016

17 (2017) 1750025

17 (2017) 1750016

17 (2017) 1750013

17 (2017) 1750030

17 (2017) 1750004 
with non-Lipschitz coefficients driven by fractional Brownian motion

Yan, L., see Sun

Yang, X., see Zhang

Yao, N., see Li

Yoon, J.-H., Kim, J.H., Choi, S.-Y. \& Han, Y., Stochastic volatility asymptotics of defaultable interest rate derivatives under a quadratic Gaussian model

Zanella, M., see Bonaccorsi
17 (2017) 1750013

17 (2017) 1750029

17 (2017) 1750012

17 (2017) 1750025

17 (2017) 1750003

17 (2017) 1750045
Zeng, Z. \& Hu, H., Weak linear representation of M-estimaton in GLMs with dependent errors

Zhang, Q., see Sun

Zhang, S., see $\mathrm{Li}$

Zhang, Y. \& Yang, X., Stochastic elastic equation driven by multiplicative multiparameter fractional noise

Zhang, Y., see Rodrigues

Zhang, Z., see Tong

Zheng, Y., see Sun
17 (2017) 1750012

17 (2017) 1750035

17 (2017) 1750037

17 (2017) 1750034

17 (2017) 1750029

17 (2017) 1750025

17 (2017) 1750033 